\title{
Compound Synchronization of Four Chaotic Complex Systems
}

\author{
Junwei Sun, ${ }^{1}$ Yi Shen, ${ }^{2}$ and Guangzhao Cui $^{1}$ \\ ${ }^{1}$ College of Electric and Information Engineering, Zhengzhou University of Light Industry, Zhengzhou 450002, China \\ ${ }^{2}$ School of Automation, Huazhong University of Science and Technology, Wuhan 430074, China \\ Correspondence should be addressed to Guangzhao Cui; cgzh@zzuli.edu.cn
}

Received 19 June 2015; Accepted 29 September 2015

Academic Editor: Xavier Leoncini

Copyright (C) 2015 Junwei Sun et al. This is an open access article distributed under the Creative Commons Attribution License, which permits unrestricted use, distribution, and reproduction in any medium, provided the original work is properly cited.

\begin{abstract}
The chaotic complex system is designed from the start of the chaotic real system. Dynamical properties of a chaotic complex system in complex space are investigated. In this paper, a compound synchronization scheme is achieved for four chaotic complex systems. According to Lyapunov stability theory and the adaptive control method, four chaotic complex systems are considered and the corresponding controllers are designed to realize the compound synchronization scheme. Four novel design chaotic complex systems are given as an example to verify the validity and feasibility of the proposed control scheme.
\end{abstract}

\section{Introduction}

Chaos synchronization proposed by Pecora and Carroll [1] has gained a lot of attractions for its potential application in many scientific and engineering fields in recent years. Until now, various types of chaotic synchronization scheme have been extensively investigated and a lot of valuable results have been gained, such as complete synchronization [1-4], antisynchronization $[5,6]$, generalized synchronization [710], phase synchronization [11], antiphase synchronization [12], lag synchronization [13, 14], partial synchronization [15], projective synchronization [16, 17], time scale synchronization [18], combination synchronization [19-21], and compound synchronization $[22,23]$.

The synchronization of a large number of real dynamical systems of physical nature has been considered in the above studies (i.e., those dynamical systems with real variables). However, there are also some nonlinear systems involving complex variables in the world. The complex Lorenz equations have been firstly investigated by Fowler et al. [24], which can extend nonlinear real systems [25] to nonlinear complex systems. Some chaotic complex systems have been proposed during the last few decades, such as the complex Chen system [26], the complex Lü systems [26], the hyperchaotic complex Lü system [27], the modified complex Lorenz system [28], and the hyperchaotic complex Lorenz system [29, 30]. It is well-known that the complex chaotic systems also have much wider potential application in many different fields of nonlinear sciences. The adoption of complex chaotic systems can increase more transmitted information to improve the security of the transmitted information in secure communication [24].

In general, the level of security is greatly due to the complexity level of the drive and response systems and the formation of the transmitted signals as well as the modulation scheme employed. Compound synchronization [22, 23] have been designed to split the transmitted signals into several parts, each part loaded in different drive systems to ensure stronger antiattack ability and antitranslated capability than those transmitted by the traditional transmission model. If we can realize compound synchronization scheme for three drive chaotic complex systems and one response chaotic complex system, then the transmitted signals are so complex and unpredictable that they may have stronger antiattack ability and antitranslated capability than those transmitted by the traditional synchronization model.

Inspired by the above discussion, in this paper, the chaotic complex system is designed from the start of the chaotic real system for the first time. Its dynamical properties such as invariance, dissipativity, equilibria, Lyapunov exponents, bifurcation diagrams, chaotic behavior, and chaotic attractors are investigated. According to the above complex dynamical 
properties, the compound synchronization scheme and the corresponding controllers are designed for three drive chaotic complex systems and one response chaotic complex system. The adoption of complex chaotic systems has been given for secure communication and the complex variables (doubling the number of variables) to increase the capacities and security of the transmitted information for the compound synchronization.

Compared with the previous work, there are two advantages which make our paper more charming and meaningful. At first, a chaotic complex system is designed from the start of a chaotic real system, which can produce more complex and unpredictable signals. Furthermore, we apply the general advantages of compound synchronization scheme [22, 23] for four real chaotic systems and extend it to four chaotic complex systems. The transmitted signal can be separated into several parts loaded in the compound system of three drive chaotic complex systems with different initial conditions to strengthen the security of secure communication. According to the above two advantages, our design scheme seems to have more valuable and advantageous than the traditional synchronization scheme, which may be helpful for the development of secure communication.

This paper is organized as follows. In Section 2, the dynamical properties of chaotic complex system are investigated, followed by the scheme of compound synchronization among four chaotic complex systems which is realized in Section 3. In Section 4, compound synchronization of four identical chaotic complex systems is given as an example. Finally, our concluding remarks are gained in Section 5.

\section{Basic Properties}

Referring to chaotic dynamics in the previous papers, some complex systems have been proposed from the start of chaotic real systems counterparts. In this paper, the novel chaotic complex system is designed from the start of the chaotic real system counterpart [31]. The chaotic real system counterpart [31] is

$$
\begin{aligned}
& \dot{x}=a y z, \\
& \dot{y}=1+b x, \\
& \dot{z}=x^{2}+c z,
\end{aligned}
$$

where $a, b$, and $c$ are three constants and $x, y$, and $z$ are three state variables. When $a=1, b=-4$, and $c=-1$, the nonlinear system (1) is chaotic as in Figure 1.

Our chaotic complex system is proposed for the first time in the form of

$$
\begin{aligned}
& \dot{w}_{1}=a w_{2} w_{3}, \\
& \dot{w}_{2}=1+b w_{1}, \\
& \dot{w}_{3}=w_{1} \bar{w}_{1}+c w_{3},
\end{aligned}
$$

where $a, b$, and $c$ are three constants of the nonlinear complex system (2), $w_{1}=u_{1}+j u_{2}, w_{2}=u_{3}+j u_{4}$ are complex variables, $j=\sqrt{-1}$, and $w_{3}=u_{5}$ is a real variable.

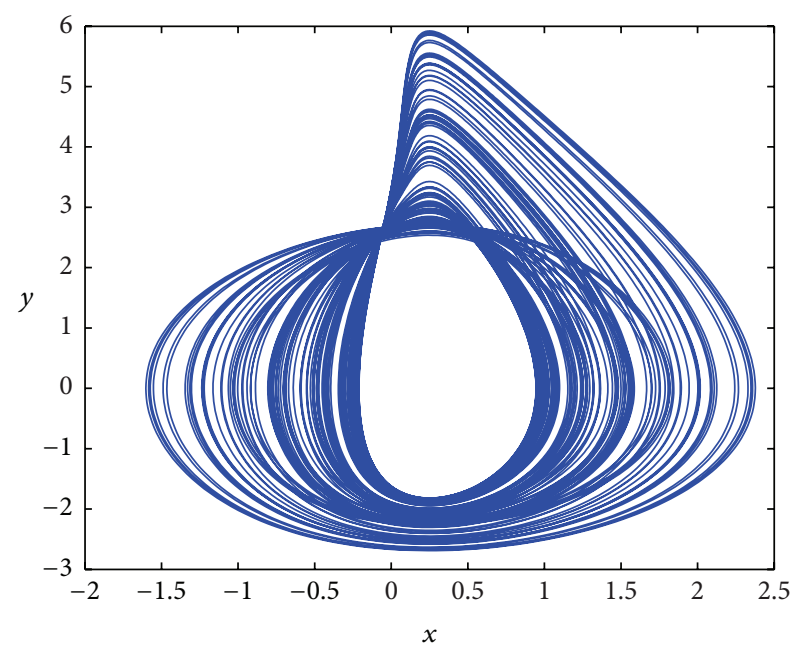

FIGURE 1: 2D projection of system (1), $x$ versus $y$.

The real system version of (2) can be represented as follows:

$$
\begin{aligned}
& \dot{u}_{1}=a u_{3} u_{5}, \\
& \dot{u}_{2}=a u_{4} u_{5}, \\
& \dot{u}_{3}=1+b u_{1}, \\
& \dot{u}_{4}=b u_{2}, \\
& \dot{u}_{5}=u_{1}^{2}+u_{2}^{2}+c u_{5} .
\end{aligned}
$$

2.1. Symmetry and Invariance. Nonlinear system (3) is symmetric about the $u_{1}, u_{3}$, and $u_{5}$ plane, which implies that it is invariant for the transformation of $\left(u_{1}, u_{2}, u_{3}, u_{4}, u_{5}\right) \rightarrow$ $\left(u_{1},-u_{2}, u_{3},-u_{4}, u_{5}\right)$. Therefore, if $\left(u_{1}, u_{2}, u_{3}, u_{4}, u_{5}\right)$ is a solution of nonlinear system (3), then $\left(u_{1},-u_{2}, u_{3},-u_{4}, u_{5}\right)$ is also a solution of the same system.

2.2. Dissipation. The divergence of nonlinear system (3) is written by

$$
\nabla \cdot F=\sum_{i=1}^{5} \frac{\partial \dot{u}_{i}}{\partial u_{i}}=c
$$

if there is a constant $c<0$, then the nonlinear system (3) is a dissipative system, which can converge by the exponential convergence form.

2.3. Equilibria. The equilibrium points of nonlinear system (3) can be gained by

$$
\begin{aligned}
a u_{3} u_{5} & =0, \\
a u_{4} u_{5} & =0, \\
1+b u_{1} & =0, \\
b u_{2} & =0, \\
u_{1}^{2}+u_{2}^{2}+c u_{5} & =0 .
\end{aligned}
$$


Obviously, from the nonlinear system (5), we can easily obtain that the equilibrium point of the nonlinear system (5) is $\left(-1 / b, 0,0,0,-1 / b^{2} c\right)$.

2.4. Lyapunov Exponents. Nonlinear system (3) can be described by the following vector notation form:

$$
\dot{\Psi}(t)=\Phi(\Psi(t) ; \xi),
$$

where $\Psi(t)=\left(\psi_{1}, \psi_{2}, \ldots, \psi_{5}\right)^{T}, \Phi=\left(\phi_{1}, \phi_{2}, \ldots, \phi_{5}\right)^{T}, \xi$ is a set of parameters, and $(\cdot)^{T}$ stands for transpose. The linearized equations defining small deviations $\delta \Psi$ from the trajectory $\Psi(t)$ are written by

$$
\delta \dot{\Psi}(t)=L_{i j}(\Psi(t) ; \xi) \delta \Psi,
$$

where $L_{i j}=\partial \phi_{i} / \partial \psi_{j}(i, j=1,2,3,4,5)$ is the Jacobian matrix in the following form:

$$
L_{i j}=\left(\begin{array}{ccccc}
0 & 0 & a u_{5} & 0 & a u_{3} \\
0 & 0 & 0 & a u_{5} & a u_{4} \\
b & 0 & 0 & 0 & 0 \\
0 & b & 0 & 0 & 0 \\
2 u_{1} & 2 u_{2} & 0 & 0 & c
\end{array}\right) .
$$

The Lyapunov exponents $\lambda_{i}$ of the nonlinear system (3) are gained by

$$
\lambda_{i}=\lim _{t \rightarrow \infty} \frac{1}{t} \log \frac{\delta \psi_{i}(t)}{\delta \psi_{i}(0)} .
$$

To compute the value of $\lambda_{i}$, we numerically solve (6) and (7) simultaneously by a simple Runge-Kutta algorithm of order 4 . The abundant chaotic behavior for this nonlinear system (3) can also be given by plotting the separation of two nearby trajectories. The Lyapunov exponents of nonlinear system (3) are given for the reasonable initial values in Figures $2-4$. Fix $b=-4, c=-1$, and vary $a$; hyperchaotic attractors exist for $a \in(0,1.18], a \in(1.21,1.26], a \in(1.32,1.47]$, and chaotic attractors for $a \in(1.18,1.21], a \in(1.26,1.32]$ in Figure 2. Fix $a=1, c=-1$, and vary $b$; hyperchaotic attractors exist for $b \in(-20,0]$ in Figure 3. Fix $a=1, b=-4$, and vary $c$; hyperchaotic attractors exist for $c \in(-1.76,-0.5]$ in Figure 4 .

2.5. Bifurcation Diagrams. In this subsection, we present bifurcation diagrams of the parameters of system (3) which show other signs that demonstrate its dynamics. These diagrams are plotted in Figure 5. It is clear that the dynamics of system (3) are similar to those obtained in Section 2.4 using Lyapunov exponents.

2.6. Chaotic Attractors. In the section, the parameters are chosen as $a=2, b=1$, and $c=-1$; the initial values are assumed as $u_{1}(0)=2, u_{2}(0)=4, u_{3}(0)=4, u_{4}(0)=3$, and $u_{5}(0)=3$, and the complex nonlinear system (3) is chaotic. The chaotic attractors of system (3) for the same choice of the parameters and initial values are shown in Figures 6-9 in $\left(u_{1}, u_{3}\right),\left(u_{2}, u_{3}\right),\left(u_{2}, u_{4}\right)$, and $\left(u_{3}, u_{5}\right)$ planes, respectively.

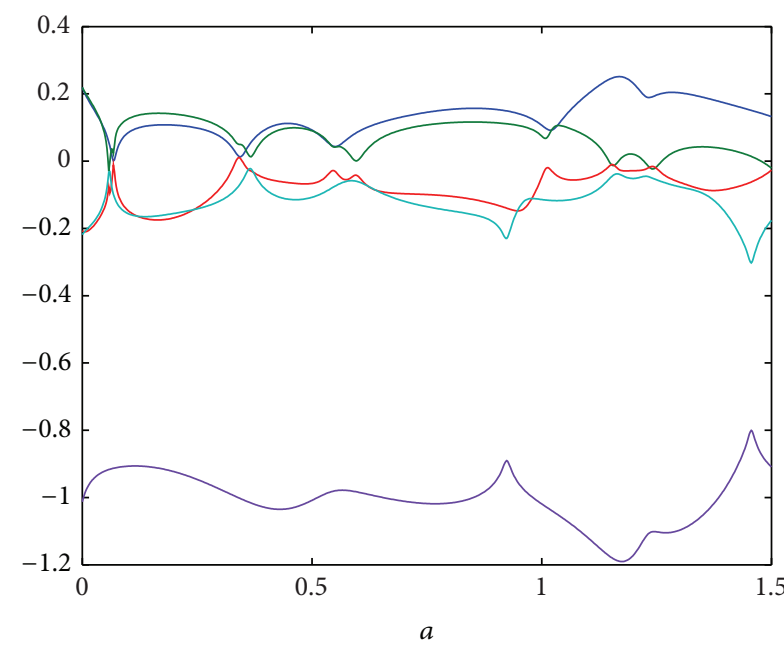

FIGURE 2: Lyapunov exponents of system (3) for $a$.

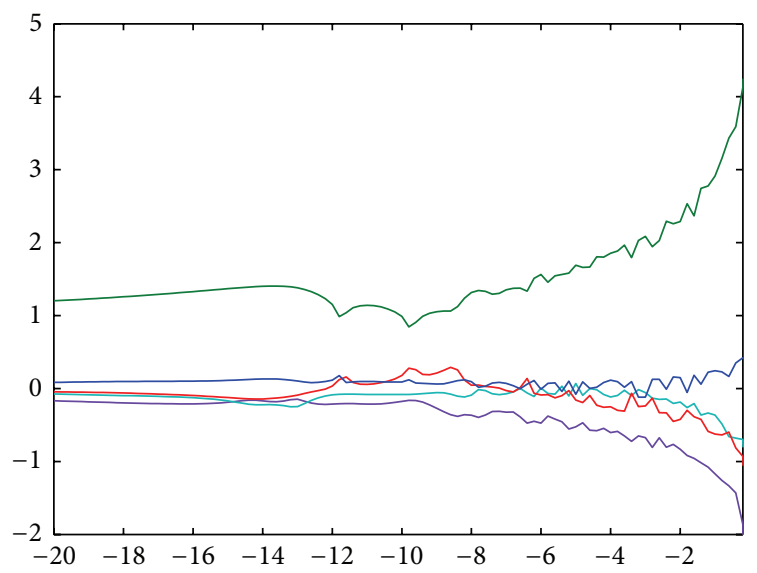

Figure 3: Lyapunov exponents of system (3) for $b$.

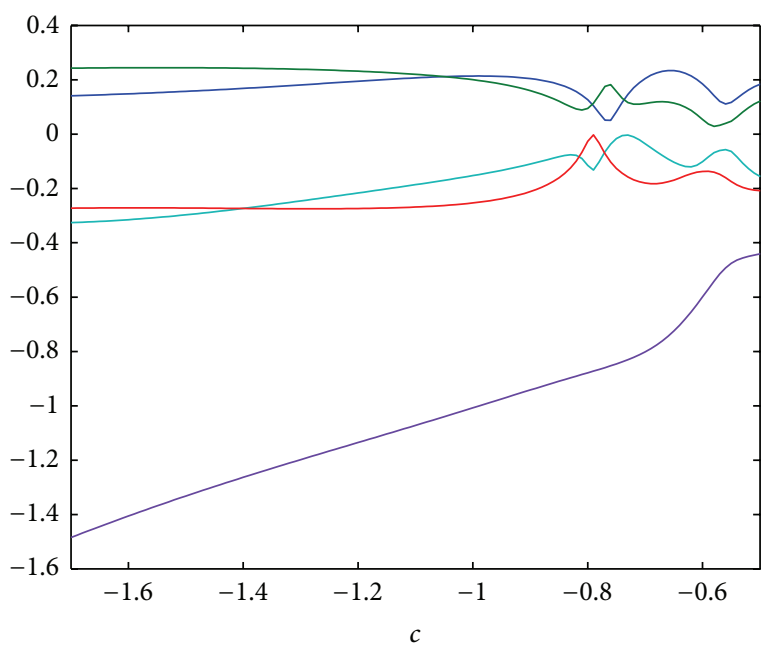

FIGURE 4: Lyapunov exponents of system (3) for $c$. 


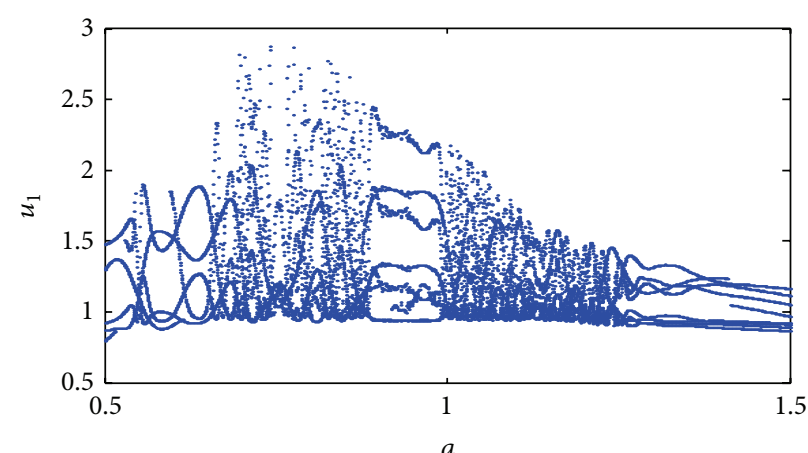

(a)

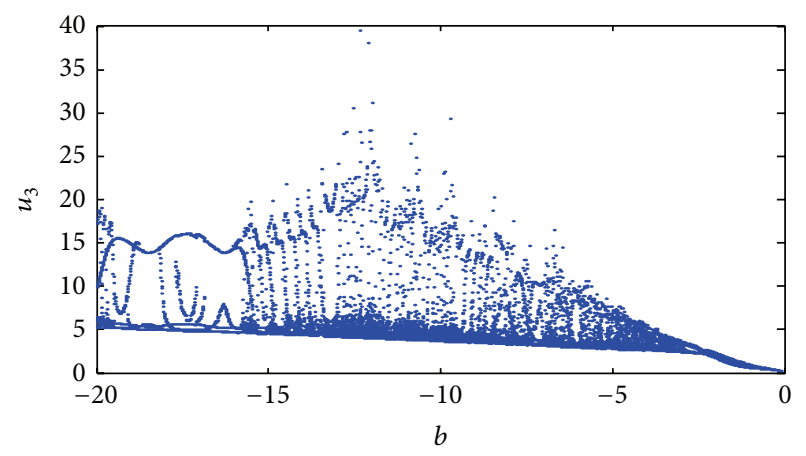

(c)

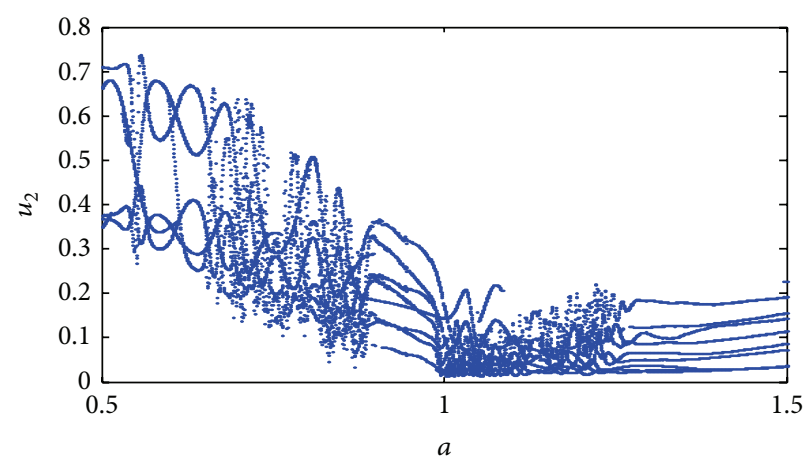

(b)

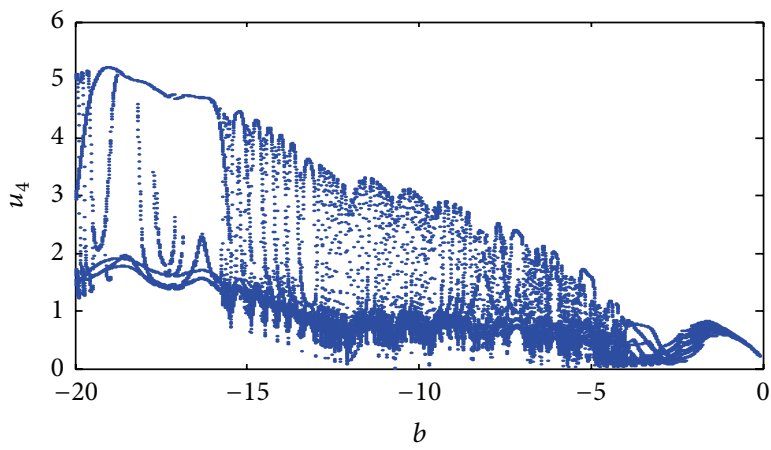

(d)

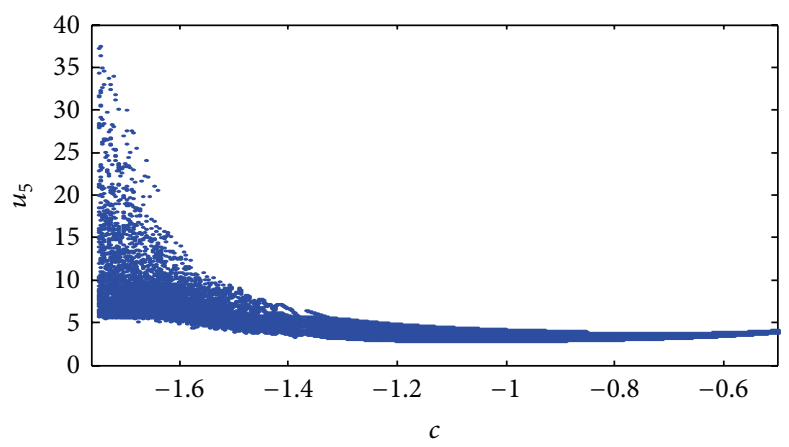

(e)

FIGURE 5: Bifurcation diagrams.

\section{Compound Synchronization}

In the section, the scheme of compound synchronization is investigated, and we assume there are three drive complex systems and one response complex system.

3.1. Model and Preliminaries. Consider three drive chaotic complex nonlinear systems in the following form:

$$
\begin{gathered}
\dot{x}_{11}(t)=F_{11}\left(x_{1}(t)\right) P_{1}+f_{11}\left(x_{1}(t)\right), \\
\dot{x}_{12}(t)=F_{12}\left(x_{1}(t)\right) P_{1}+f_{12}\left(x_{1}(t)\right), \\
\vdots \\
\dot{x}_{1 n}(t)=F_{1 n}\left(x_{1}(t)\right) P_{1}+f_{1 n}\left(x_{1}(t)\right) ;
\end{gathered}
$$

$$
\begin{aligned}
& \dot{x}_{21}(t)=F_{21}\left(x_{2}(t)\right) P_{2}+f_{21}\left(x_{2}(t)\right), \\
& \dot{x}_{22}(t)=F_{22}\left(x_{2}(t)\right) P_{2}+f_{22}\left(x_{2}(t)\right),
\end{aligned}
$$

$$
\begin{aligned}
& \dot{x}_{2 n}(t)=F_{2 n}\left(x_{2}(t)\right) P_{2}+f_{2 n}\left(x_{2}(t)\right) ; \\
& \dot{x}_{31}(t)=F_{31}\left(x_{3}(t)\right) P_{3}+f_{31}\left(x_{3}(t)\right), \\
& \dot{x}_{32}(t)=F_{32}\left(x_{3}(t)\right) P_{3}+f_{32}\left(x_{3}(t)\right),
\end{aligned}
$$




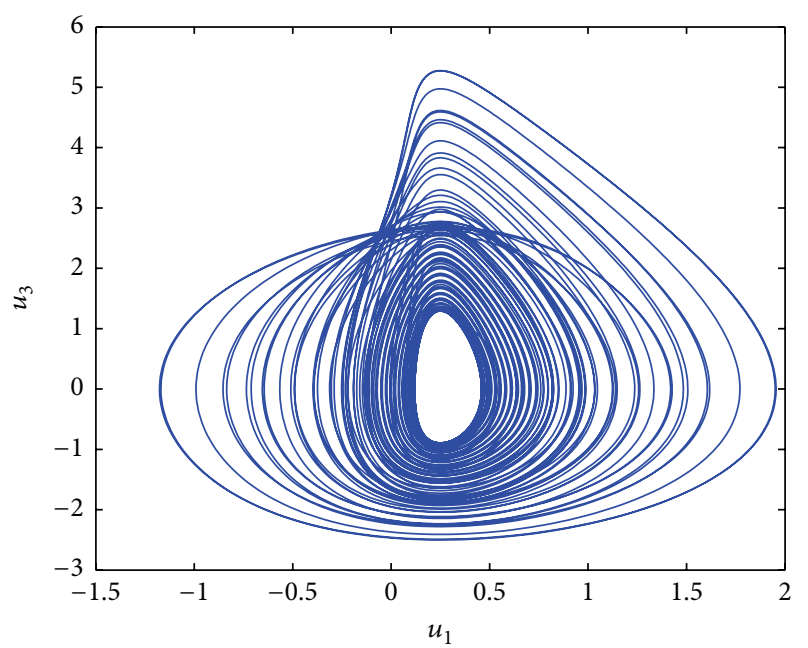

FIGURE 6: 2D projection of system (3), $u_{1}$ versus $u_{3}$.

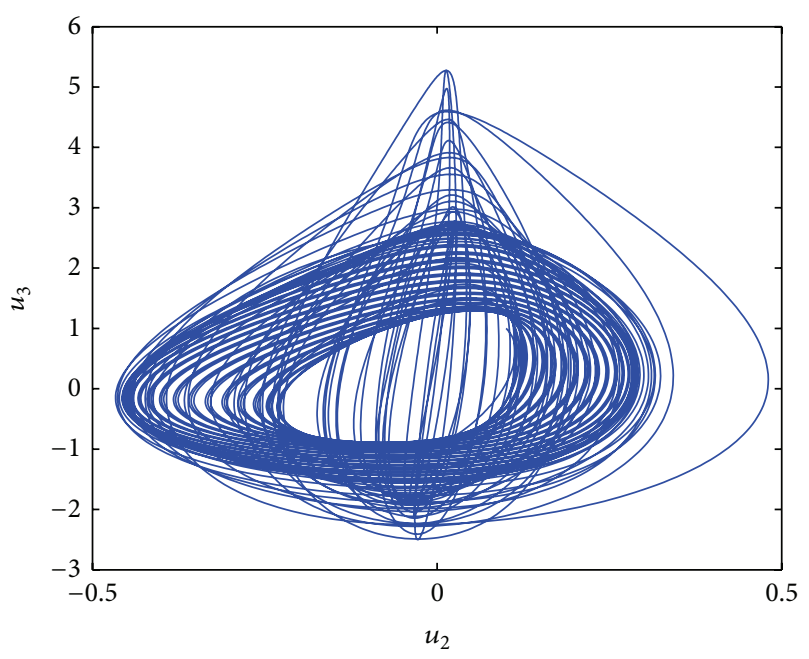

FIGURE 7: 2D projection of system (3), $u_{2}$ versus $u_{3}$.

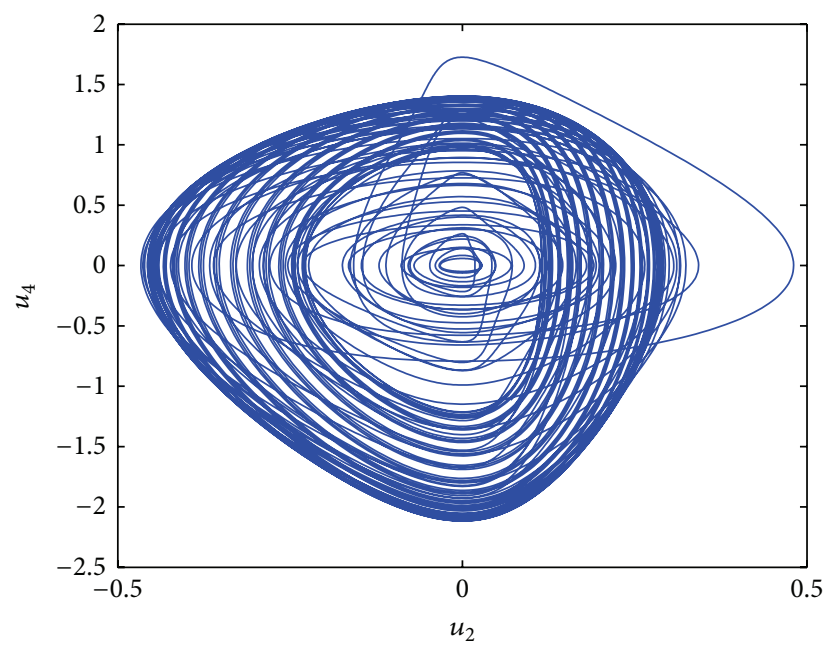

Figure 8: 2D projection of system (3), $u_{2}$ versus $u_{4}$.

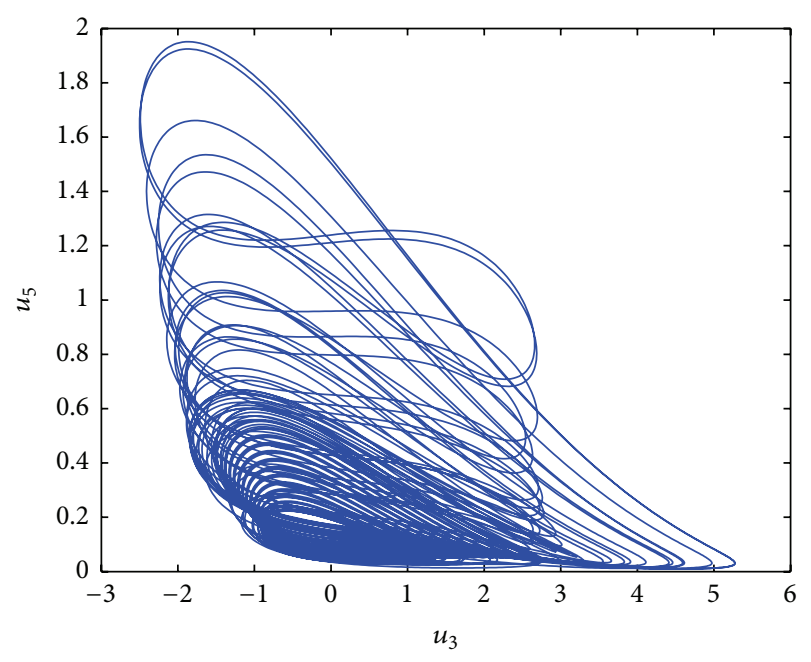

FIGURE 9: 2D projection of system (3), $u_{3}$ versus $u_{5}$.

where $x_{1}=\left(x_{11}, x_{12}, \ldots, x_{1 n}\right)^{T}, x_{2}=\left(x_{21}, x_{22}, \ldots, x_{2 n}\right)^{T}$, and $x_{3}=\left(x_{31}, x_{32}, \ldots, x_{3 n}\right)^{T}$ are three state complex vectors and $x_{1}=x_{1}^{r}+j x_{1}^{i}, x_{2}=x_{2}^{r}+j x_{2}^{i}, x_{3}=x_{3}^{r}+j x_{3}^{i}$. Assume $x_{11}=u_{11}+j u_{12}, x_{12}=u_{13}+j u_{14}, \ldots, x_{1 n}=u_{12 n-1}+j u_{12 n}$, $x_{21}=u_{21}+j u_{22}, x_{22}=u_{23}+j u_{24}, \ldots, x_{2 n}=u_{22 n-1}+j u_{22 n}$, $x_{31}=u_{31}+j u_{32}, x_{32}=u_{33}+j u_{34}, \ldots, x_{3 n}=u_{32 n-1}+j u_{32 n}$, and then $x_{1}^{r}=\left(u_{11}, u_{13}, \ldots, u_{12 n-1}\right)^{T}, x_{1}^{i}=\left(u_{12}, u_{14}, \ldots, u_{12 n}\right)^{T}$, $x_{2}^{r}=\left(u_{21}, u_{23}, \ldots, u_{22 n-1}\right)^{T}, x_{2}^{i}=\left(u_{22}, u_{24}, \ldots, u_{22 n}\right)^{T}$, $x_{3}^{r}=\left(u_{31}, u_{33}, \ldots, u_{32 n-1}\right)^{T}, x_{3}^{i}=\left(u_{32}, u_{34}, \ldots, u_{32 n}\right)^{T} . F_{1}\left(x_{1}\right)$, $F_{2}\left(x_{2}\right)$, and $F_{3}\left(x_{3}\right)$ are three $n \times n$ complex matrices and the elements of complex matrix are functions of state complex variables; $F_{1}=\left(F_{11}, F_{12}, \ldots, F_{1 n}\right)^{T}, F_{2}=\left(F_{21}, F_{22}, \ldots, F_{2 n}\right)^{T}$, and $F_{3}=\left(F_{31}, F_{32}, \ldots, F_{3 n}\right)^{T} \cdot f_{1}=\left(f_{11}, f_{12}, \ldots, f_{1 n}\right)^{T}$, $f_{2}=\left(f_{21}, f_{22}, \ldots, f_{2 n}\right)^{T}$, and $f_{3}=\left(f_{31}, f_{32}, \ldots, f_{3 n}\right)^{T}$ are three vectors of complex nonlinear functions, $P_{1}=$ $\left(p_{11}, p_{12}, \ldots, p_{1 n}\right)^{T}, P_{2}=\left(p_{21}, p_{22}, \ldots, p_{2 n}\right)^{T}$, and $P_{3}=$ $\left(p_{31}, p_{32}, \ldots, p_{3 n}\right)^{T}$ are three $n \times 1$ real (or complex) vectors of system parameters, and superscripts $r$ and $i$ denote the real and imaginary parts of the state complex vectors $x_{1}, x_{2}$, and $x_{3}$, respectively.

The response chaotic complex system can be described by

$$
\begin{gathered}
\dot{y}_{1}=G_{1}(y) Q+g_{1}(y)+v_{1}+j v_{2}, \\
\dot{y}_{2}=G_{2}(y) Q+g_{2}(y)+v_{3}+j v_{4}, \\
\vdots \\
\dot{y}_{n}=G_{n}(y) Q+g_{n}(y)+v_{2 n-1}+j v_{2 n},
\end{gathered}
$$

where $y=\left(y_{1}, y_{2}, \ldots, y_{n}\right)^{T}$ is a state complex vector and $y=y^{r}+j y^{i}$. Assume $y_{1}=u_{1}^{\prime}+j u_{2}^{\prime}, y_{2}=u_{3}^{\prime}+$ $j u_{4}^{\prime}, \ldots, y_{n}=u_{2 n-1}^{\prime}+j u_{2 n}^{\prime}$, and then $y^{r}=\left(u_{1}^{\prime}, u_{3}^{\prime}, \ldots, u_{2 n-1}^{\prime}\right)$, $y^{i}=\left(u_{2}^{\prime}, u_{4}^{\prime}, \ldots, u_{2 n}^{\prime}\right)^{T} . G(y)$ is $n \times n$ complex matrix and the elements of it are functions of state complex variables; $G=\left(G_{1}, G_{2}, \ldots, G_{n}\right)^{T} \cdot g=\left(g_{1}, g_{2}, \ldots, g_{n}\right)^{T}$ is a vector of complex nonlinear functions, $Q=\left(q_{1}, q_{2}, \ldots, q_{n}\right)^{T}$ is a 
$n \times 1$ real (or complex) vector of system parameters, and superscripts $r$ and $i$ denote the real and imaginary parts of the state complex vector $y$, respectively. $v^{r}=\left(v_{1}, v_{3}, \ldots, v_{2 n-1}\right)^{T}$, $v^{i}=\left(v_{2}, v_{4}, \ldots, v_{2 n}\right)^{T}$ are the control laws to be designed.

Definition 1. If there exist three constant diagonal real matrices $A, B, C$, such that

$$
\lim _{t \rightarrow \infty} e=\lim _{t \rightarrow \infty}\left[A X_{1}\left(B X_{2}+C X_{3}\right)-Y\right]=0
$$

can hold true, then three drive complex systems (10), (11), and (12) realized the compound synchronization with the response complex system (13), where the constant matrices $A$, $B, C$ are referred to as the real scaling matrices, four state vectors $x_{1}=\left(x_{11}, x_{12}, \ldots, x_{1 n}\right)^{T}, x_{2}=\left(x_{21}, x_{22}, \ldots, x_{2 n}\right)^{T}, x_{3}=$ $\left(x_{31}, x_{32}, \ldots, x_{3 n}\right)^{T}$, and $y=\left(y_{1}, y_{2}, \ldots, y_{n}\right)^{T}$ are described by four diagonal matrices $X_{1}=\operatorname{diag}\left(x_{11}, x_{12}, \ldots, x_{1 n}\right), X_{2}=$ $\operatorname{diag}\left(x_{21}, x_{22}, \ldots, x_{2 n}\right), X_{3}=\operatorname{diag}\left(x_{31}, x_{32}, \ldots, x_{3 n}\right)$, and $Y=$ $\operatorname{diag}\left(y_{1}, y_{2}, \ldots, y_{n}\right)$.

Remark 2. If one of these two constant diagonal real matrices $B \neq 0$ or $C \neq 0$, then the compound synchronization will be changed into a novel type of function projective synchronization between two different chaotic complex systems. If the scaling matrix $A=0$ or $B=C=0$, then the compound synchronization of the chaotic complex systems will be changed into a chaos control problem of the chaotic system.

Remark 3. Definition 1 shows that the compound drive system consists of more chaotic complex systems. In addition, these drive systems in the compound synchronization can be identical or different. At last, three constant diagonal real matrices can be extended to three constant diagonal complex matrices.

Remark 4. It is worth mentioning that the compound synchronization scheme is different from the previously investigated combination synchronization scheme of three chaotic systems [20,21]. The combination system of two drive systems has achieved the combination synchronization scheme with one response system; however, the compound system of three chaotic complex systems has realized the compound synchronization with one response system in our scheme. The transmitted signal can be separated into several parts loaded in the mixed system of three different drive systems or three same drive systems with different initial conditions to increase antiattack ability and antitranslated capability.

Remark 5. In the compound synchronization scheme [22, 23], some studies have mostly been confined within four chaotic real systems, which are three drive real systems and one response real system. Four chaotic systems are real in the known results on compound synchronization; however, four chaotic systems are complex in our scheme. The adoption of complex chaotic systems has been given for secure communication and the complex variables (doubling the number of variables) to increase the capacities and security of the transmitted information for the compound synchronization.
For the convenience of our discussions, we assume $A=\operatorname{diag}\left(a_{1}, a_{2}, \ldots, a_{n}\right), B=\operatorname{diag}\left(b_{1}, b_{2}, \ldots, b_{n}\right), C=$ $\operatorname{diag}\left(c_{1}, c_{2}, \ldots, c_{n}\right)$ in our synchronization scheme.

3.2. Main Result. In the section, the compound synchronization between three drive complex systems (10), (11), and (12) and one response complex system (13) is investigated.

Theorem 6. If nonlinear controller is designed as the form

$$
\begin{aligned}
v_{k} & =v_{2 k-1}+j v_{2 k}=a_{k}\left[F_{1 k}^{r}\left(x_{1}\right) P_{1}+f_{1 k}^{r}\left(x_{1}\right)\right]\left(b_{k} x_{2 k}^{r}\right. \\
& \left.+c_{k} x_{3 k}^{r}\right)-a_{k}\left[F_{1 k}^{i}\left(x_{1}\right) P_{1}+f_{1 k}^{i}\left(x_{1}\right)\right]\left(b_{k} x_{2 k}^{i}\right. \\
& \left.+c_{k} x_{3 k}^{i}\right)+a_{k} x_{1 k}^{r}\left\{b_{k}\left[F_{2 k}^{r}\left(x_{2}\right) P_{2}+f_{2 k}^{r}\left(x_{2}\right)\right]\right. \\
& \left.+c_{k}\left[F_{3 k}^{r}\left(x_{3}\right) P_{3}+f_{3 k}^{r}\left(x_{3}\right)\right]\right\} \\
& -a_{k} x_{1 k}^{i}\left\{b_{k}\left[F_{2 k}^{i}\left(x_{2}\right) P_{2}+f_{2 k}^{i}\left(x_{2}\right)\right]\right. \\
& \left.+c_{k}\left[F_{3 k}^{i}\left(x_{3}\right) P_{3}+f_{3 k}^{i}\left(x_{3}\right)\right]\right\}-G_{k}^{r}(y) Q-g_{k}^{r}(y) \\
& +l e_{k}^{r}+j\left\{a _ { k } ( F _ { 1 k } ^ { r } ( x _ { 1 } ) P _ { 1 } + f _ { 1 k } ^ { r } ( x _ { 1 } ) ) \left(b_{k} x_{2 k}^{i}\right.\right. \\
& \left.+c_{k} x_{3 k}^{i}\right)+a_{k}\left(F_{1 k}^{i}\left(x_{1}\right) P_{1}+f_{1 k}^{i}\left(x_{1}\right)\right)\left(b_{k} x_{2 k}^{r}\right. \\
& \left.+c_{k} x_{3 k}^{r}\right)+a_{k} x_{1 k}^{r}\left[b_{k}\left(F_{2 k}^{i}\left(x_{2}\right) P_{2}+f_{2 k}^{i}\left(x_{2}\right)\right)\right. \\
& \left.+c_{k}\left(F_{3 k}^{i}\left(x_{3}\right) P_{3}+f_{3 k}^{i}\left(x_{3}\right)\right)\right] \\
& +a_{k} x_{1 k}^{i}\left[b_{k}\left(F_{2 k}^{r}\left(x_{2}\right) P_{2}+f_{2 k}^{r}\left(x_{2}\right)\right)\right. \\
& \left.+c_{k}\left(F_{3 k}^{r}\left(x_{3}\right) P_{3}+f_{3 k}^{r}\left(x_{3}\right)\right)\right]-G_{k}^{i}(y) Q-g_{k}^{i}(y) \\
& \left.+l e_{k}^{i}\right\},
\end{aligned}
$$

then the compound synchronization between three drive systems (10), (11), and (12) and one response system (13) is achieved, where l $>0$ is a constant.

Proof. From the four chaotic complex systems, the synchronization error can be defined as follows:

$$
e_{k}=e_{k}^{r}+j e_{k}^{i}=a_{k} x_{1 k}\left(b_{k} x_{2 k}+c_{k} x_{3 k}\right)-y_{k},
$$

where $k=1,2, \ldots, n$. The derivative of the error system (16) can be written as

$$
\begin{aligned}
\dot{e}_{k} & =a_{k} \dot{x}_{1 k}\left(b_{k} x_{2 k}+c_{k} x_{3 k}\right)+a_{k} x_{1 k}\left(b_{k} \dot{x}_{2 k}+c_{k} \dot{x}_{3 k}\right) \\
& -y_{k}=a_{k}\left[F_{1 k}^{r}\left(x_{1}\right) P_{1}+f_{1 k}^{r}\left(x_{1}\right)+j\left(F_{1 k}^{i}\left(x_{1}\right) P_{1}\right.\right. \\
& \left.\left.+f_{1 k}^{i}\left(x_{1}\right)\right)\right]\left[b_{k}\left(x_{2 k}^{r}+j x_{2 k}^{i}\right)+c_{k}\left(x_{3 k}^{r}+j x_{3 k}^{i}\right)\right] \\
& +a_{k}\left(x_{1 k}^{r}+j x_{1 k}^{i}\right)\left\{b _ { k } \left[F_{2 k}^{r}\left(x_{2}\right) P_{2}+f_{2 k}^{r}\left(x_{2}\right)\right.\right. \\
& \left.+j\left(F_{2 k}^{i}\left(x_{2}\right) P_{2}+f_{2 k}^{i}\left(x_{2}\right)\right)\right]+c_{k}\left[F_{3 k}^{r}\left(x_{3}\right) P_{3}\right. \\
& \left.\left.+f_{3 k}^{r}\left(x_{3}\right)+j\left(F_{3 k}^{i}\left(x_{3}\right) P_{3}+f_{3 k}^{i}\left(x_{3}\right)\right)\right]\right\}-G_{k}^{r}(y) \\
& \cdot Q-g_{k}^{r}(y)-v_{2 k-1}-j\left[G_{k}^{i}(y) Q+g_{k}^{i}(y)+v_{2 k}\right] .
\end{aligned}
$$


By the further computation, we get

$$
\begin{aligned}
\dot{e}_{k} & =a_{k}\left(F_{1 k}^{r}\left(x_{1}\right) P_{1}+f_{1 k}^{r}\left(x_{1}\right)\right)\left(b_{k} x_{2 k}^{r}+c_{k} x_{3 k}^{r}\right) \\
& -a_{k}\left(F_{1 k}^{i}\left(x_{1}\right) P_{1}+f_{1 k}^{i}\left(x_{1}\right)\right)\left(b_{k} x_{2 k}^{i}+c_{k} x_{3 k}^{i}\right) \\
& \cdot j\left[a_{k}\left(F_{1 k}^{r}\left(x_{1}\right) P_{1}+f_{1 k}^{r}\left(x_{1}\right)\right)\left(b_{k} x_{2 k}^{i}+c_{k} x_{3 k}^{i}\right)\right. \\
& \left.+a_{k}\left(F_{1 k}^{i}\left(x_{1}\right) P_{1}+f_{1 k}^{i}\left(x_{1}\right)\right)\left(b_{k} x_{2 k}^{r}+c_{k} x_{3 k}^{r}\right)\right] \\
& +a_{k} x_{1 k}^{r}\left\{b_{k}\left[F_{2 k}^{r}\left(x_{2}\right) P_{2}+f_{2 k}^{r}\left(x_{2}\right)\right]\right. \\
& \left.+c_{k}\left[F_{3 k}^{r}\left(x_{3}\right) P_{3}+f_{3 k}^{r}\left(x_{3}\right)\right]\right\} \\
& -a_{k} x_{1 k}^{i}\left\{b_{k}\left[F_{2 k}^{i}\left(x_{2}\right) P_{2}+f_{2 k}^{i}\left(x_{2}\right)\right]\right. \\
& \left.+c_{k}\left[F_{3 k}^{i}\left(x_{3}\right) P_{3}+f_{3 k}^{i}\left(x_{3}\right)\right]\right\} \\
& +j a_{k} x_{1 k}^{r}\left[b_{k}\left(F_{2 k}^{i}\left(x_{2}\right) P_{2}+f_{2 k}^{i}\left(x_{2}\right)\right)\right. \\
& \left.+c_{k}\left(F_{3 k}^{i}\left(x_{3}\right) P_{3}+f_{3 k}^{i}\left(x_{3}\right)\right)\right] \\
& +a_{k} x_{1 k}^{i}\left[b_{k}\left(F_{2 k}^{r}\left(x_{2}\right) P_{2}+f_{2 k}^{r}\left(x_{2}\right)\right)\right. \\
& \left.+c_{k}\left(F_{3 k}^{r}\left(x_{3}\right) P_{3}+f_{3 k}^{r}\left(x_{3}\right)\right)\right]-G_{k}^{r}(y) Q-g_{k}^{r}(y) \\
& -v_{2 k-1}-j\left[G_{k}^{i}(y) Q+g_{k}^{i}(y)+v_{2 k}\right] .
\end{aligned}
$$

Hence,

$$
\begin{aligned}
\dot{e}_{k} & =a_{k}\left[F_{1 k}^{r}\left(x_{1}\right) P_{1}+f_{1 k}^{r}\left(x_{1}\right)\right]\left(b_{k} x_{2 k}^{r}+c_{k} x_{3 k}^{r}\right) \\
& -a_{k}\left[F_{1 k}^{i}\left(x_{1}\right) P_{1}+f_{1 k}^{i}\left(x_{1}\right)\right]\left(b_{k} x_{2 k}^{i}+c_{k} x_{3 k}^{i}\right) \\
& +a_{k} x_{1 k}^{r}\left\{b_{k}\left[F_{2 k}^{r}\left(x_{2}\right) P_{2}+f_{2 k}^{r}\left(x_{2}\right)\right]\right. \\
& \left.+c_{k}\left[F_{3 k}^{r}\left(x_{3}\right) P_{3}+f_{3 k}^{r}\left(x_{3}\right)\right]\right\} \\
& -a_{k} x_{1 k}^{i}\left\{b_{k}\left[F_{2 k}^{i}\left(x_{2}\right) P_{2}+f_{2 k}^{i}\left(x_{2}\right)\right]\right. \\
& \left.+c_{k}\left[F_{3 k}^{i}\left(x_{3}\right) P_{3}+f_{3 k}^{i}\left(x_{3}\right)\right]\right\}-G_{k}^{r}(y) Q-g_{k}^{r}(y) \\
& -v_{2 k-1}+j\left\{a _ { k } ( F _ { 1 k } ^ { r } ( x _ { 1 } ) P _ { 1 } + f _ { 1 k } ^ { r } ( x _ { 1 } ) ) \left(b_{k} x_{2 k}^{i}\right.\right. \\
& \left.+c_{k} x_{3 k}^{i}\right)+a_{k}\left(F_{1 k}^{i}\left(x_{1}\right) P_{1}+f_{1 k}^{i}\left(x_{1}\right)\right)\left(b_{k} x_{2 k}^{r}\right. \\
& \left.+c_{k} x_{3 k}^{r}\right)+a_{k} x_{1 k}^{r}\left[b_{k}\left(F_{2 k}^{i}\left(x_{2}\right) P_{2}+f_{2 k}^{i}\left(x_{2}\right)\right)\right. \\
& \left.+c_{k}\left(F_{3 k}^{i}\left(x_{3}\right) P_{3}+f_{3 k}^{i}\left(x_{3}\right)\right)\right] \\
& +a_{k} x_{1 k}^{i}\left[b_{k}\left(F_{2 k}^{r}\left(x_{2}\right) P_{2}+f_{2 k}^{r}\left(x_{2}\right)\right)\right. \\
& \left.+c_{k}\left(F_{3 k}^{r}\left(x_{3}\right) P_{3}+f_{3 k}^{r}\left(x_{3}\right)\right)\right]-G_{k}^{i}(y) Q-g_{k}^{i}(y) \\
& \left.-v_{2 k}\right\} .
\end{aligned}
$$

Separating real and imaginary parts in (19), one can obtain

$$
\begin{aligned}
\dot{e}_{k}^{r} & =a_{k}\left[F_{1 k}^{r}\left(x_{1}\right) P_{1}+f_{1 k}^{r}\left(x_{1}\right)\right]\left(b_{k} x_{2 k}^{r}+c_{k} x_{3 k}^{r}\right) \\
& -a_{k}\left[F_{1 k}^{i}\left(x_{1}\right) P_{1}+f_{1 k}^{i}\left(x_{1}\right)\right]\left(b_{k} x_{2 k}^{i}+c_{k} x_{3 k}^{i}\right) \\
& +a_{k} x_{1 k}^{r}\left\{b_{k}\left[F_{2 k}^{r}\left(x_{2}\right) P_{2}+f_{2 k}^{r}\left(x_{2}\right)\right]\right. \\
& \left.+c_{k}\left[F_{3 k}^{r}\left(x_{3}\right) P_{3}+f_{3 k}^{r}\left(x_{3}\right)\right]\right\} \\
& -a_{k} x_{1 k}^{i}\left\{b_{k}\left[F_{2 k}^{i}\left(x_{2}\right) P_{2}+f_{2 k}^{i}\left(x_{2}\right)\right]\right. \\
& \left.+c_{k}\left[F_{3 k}^{i}\left(x_{3}\right) P_{3}+f_{3 k}^{i}\left(x_{3}\right)\right]\right\}-G_{k}^{r}(y) Q-g_{k}^{r}(y) \\
& -v_{2 k-1}, \\
\dot{e}_{k}^{i} & =a_{k}\left(F_{1 k}^{r}\left(x_{1}\right) P_{1}+f_{1 k}^{r}\left(x_{1}\right)\right)\left(b_{k} x_{2 k}^{i}+c_{k} x_{3 k}^{i}\right) \\
& +a_{k}\left(F_{1 k}^{i}\left(x_{1}\right) P_{1}+f_{1 k}^{i}\left(x_{1}\right)\right)\left(b_{k} x_{2 k}^{r}+c_{k} x_{3 k}^{r}\right) \\
& +a_{k} x_{1 k}^{r}\left[b_{k}\left(F_{2 k}^{i}\left(x_{2}\right) P_{2}+f_{2 k}^{i}\left(x_{2}\right)\right)\right. \\
& \left.+c_{k}\left(F_{3 k}^{i}\left(x_{3}\right) P_{3}+f_{3 k}^{i}\left(x_{3}\right)\right)\right] \\
& +a_{k} x_{1 k}^{i}\left[b_{k}\left(F_{2 k}^{r}\left(x_{2}\right) P_{2}+f_{2 k}^{r}\left(x_{2}\right)\right)\right. \\
& \left.+c_{k}\left(F_{3 k}^{r}\left(x_{3}\right) P_{3}+f_{3 k}^{r}\left(x_{3}\right)\right)\right]-G_{k}^{i}(y) Q-g_{k}^{i}(y) \\
& -v_{2 k} .
\end{aligned}
$$

Choose a Lyapunov function candidate as

$$
V\left(e^{r}, e^{i}\right)=\frac{1}{2} \sum_{k=1}^{n}\left[\left(e_{k}^{r}\right)^{T} e_{k}^{r}+\left(e_{k}^{i}\right)^{T} e_{k}^{i}\right],
$$

then time derivative of $V$ along the trajectory of the error systems (20) is as follows:

$$
\begin{aligned}
\dot{V} & =\sum_{k=1}^{n}\left(\dot{e}_{k}^{r}\right)^{T} e_{k}^{r}+\left(\dot{e}_{k}^{i}\right)^{T} e_{k}^{i}=\sum_{k=1}^{n}\left\{a _ { k } \left[F_{1 k}^{r}\left(x_{1}\right) P_{1}\right.\right. \\
& \left.+f_{1 k}^{r}\left(x_{1}\right)\right]\left(b_{k} x_{2 k}^{r}+c_{k} x_{3 k}^{r}\right)-a_{k}\left[F_{1 k}^{i}\left(x_{1}\right) P_{1}\right. \\
& \left.+f_{1 k}^{i}\left(x_{1}\right)\right]\left(b_{k} x_{2 k}^{i}+c_{k} x_{3 k}^{i}\right) \\
& +a_{k} x_{1 k}^{r}\left\{b_{k}\left[F_{2 k}^{r}\left(x_{2}\right) P_{2}+f_{2 k}^{r}\left(x_{2}\right)\right]\right. \\
& \left.+c_{k}\left[F_{3 k}^{r}\left(x_{3}\right) P_{3}+f_{3 k}^{r}\left(x_{3}\right)\right]\right\} \\
& -a_{k} x_{1 k}^{i}\left\{b_{k}\left[F_{2 k}^{i}\left(x_{2}\right) P_{2}+f_{2 k}^{i}\left(x_{2}\right)\right]\right. \\
& \left.+c_{k}\left[F_{3 k}^{i}\left(x_{3}\right) P_{3}+f_{3 k}^{i}\left(x_{3}\right)\right]\right\}-G_{k}^{r}(y) Q-g_{k}^{r}(y) \\
& \left.-v_{2 k-1}\right\}^{T} e_{k}^{r}+\sum_{k=1}^{n}\left\{a_{k}\left(F_{1 k}^{r}\left(x_{1}\right) P_{1}+f_{1 k}^{r}\left(x_{1}\right)\right)\right. \\
& \cdot\left(b_{k} x_{2 k}^{i}+c_{k} x_{3 k}^{i}\right)+a_{k}\left(F_{1 k}^{i}\left(x_{1}\right) P_{1}+f_{1 k}^{i}\left(x_{1}\right)\right) \\
& \cdot\left(b_{k} x_{2 k}^{r}+c_{k} x_{3 k}^{r}\right)
\end{aligned}
$$




$$
\begin{aligned}
& +a_{k} x_{1 k}^{r}\left[b_{k}\left(F_{2 k}^{i}\left(x_{2}\right) P_{2}+f_{2 k}^{i}\left(x_{2}\right)\right)\right. \\
& \left.+c_{k}\left(F_{3 k}^{i}\left(x_{3}\right) P_{3}+f_{3 k}^{i}\left(x_{3}\right)\right)\right] \\
& +a_{k} x_{1 k}^{i}\left[b_{k}\left(F_{2 k}^{r}\left(x_{2}\right) P_{2}+f_{2 k}^{r}\left(x_{2}\right)\right)\right. \\
& \left.+c_{k}\left(F_{3 k}^{r}\left(x_{3}\right) P_{3}+f_{3 k}^{r}\left(x_{3}\right)\right)\right]-G_{k}^{i}(y) Q-g_{k}^{i}(y) \\
& \left.-v_{2 k}\right\}^{T} e_{k}^{i} . \\
& =-\sum_{k=1}^{n} l\left[\left(e_{k}^{r}\right)^{T} e_{k}^{r}+\left(e_{k}^{i}\right)^{T} e_{k}^{i}\right] .
\end{aligned}
$$

Since $V$ is positive definite and $\dot{V}$ is negative semidefinite, then the response system (13) can asymptotically synchronize the compound system between the drive complex systems (10), (11), and (12), respectively. This completes the proof.

The following corollaries are easily gained from Theorem 6, and their proofs are omitted.

Corollary 7. Assume the scaling matrix $B=0$; if the nonlinear controller is designed as the form

$$
\begin{aligned}
v_{k} & =a_{k}\left[F_{1 k}^{r}\left(x_{1}\right) P_{1}+f_{1 k}^{r}\left(x_{1}\right)\right] c_{k} x_{3 k}^{r} \\
& -a_{k}\left[F_{1 k}^{i}\left(x_{1}\right) P_{1}+f_{1 k}^{i}\left(x_{1}\right)\right] c_{k} x_{3 k}^{i} \\
& +a_{k} x_{1 k}^{r} c_{k}\left[F_{3 k}^{r}\left(x_{3}\right) P_{3}+f_{3 k}^{r}\left(x_{3}\right)\right] \\
& -a_{k} x_{1 k}^{i} c_{k}\left[F_{3 k}^{i}\left(x_{3}\right) P_{3}+f_{3 k}^{i}\left(x_{3}\right)\right]-G_{k}^{r}(y) Q \\
& -g_{k}^{r}(y)+l e_{k}^{r} \\
& +j\left\{a_{k}\left[F_{1 k}^{r}\left(x_{1}\right) P_{1}+f_{1 k}^{r}\left(x_{1}\right)\right] c_{k} x_{3 k}^{i}\right. \\
& +a_{k}\left[F_{1 k}^{i}\left(x_{1}\right) P_{1}+f_{1 k}^{i}\left(x_{1}\right)\right] c_{k} x_{3 k}^{r} \\
& +a_{k} x_{1 k}^{r} c_{k}\left[F_{3 k}^{i}\left(x_{3}\right) P_{3}+f_{3 k}^{i}\left(x_{3}\right)\right] \\
& +a_{k} x_{1 k}^{i} c_{k}\left[F_{3 k}^{r}\left(x_{3}\right) P_{3}+f_{3 k}^{r}\left(x_{3}\right)\right]-G_{k}^{i}(y) Q \\
& \left.-g_{k}^{i}(y)+l e_{k}^{i}\right\},
\end{aligned}
$$

then the drive complex systems (10) and (12) will achieve a novel type of function projective synchronization with the response complex system (13), where $l>0$ is a constant.

Corollary 8. Assume the scaling matrix $C=0$; if the nonlinear controller is designed as the form

$$
\begin{aligned}
v_{k} & =a_{k}\left[F_{1 k}^{r}\left(x_{1}\right) P_{1}+f_{1 k}^{r}\left(x_{1}\right)\right] b_{k} x_{2 k}^{r} \\
& -a_{k}\left[F_{1 k}^{i}\left(x_{1}\right) P_{1}+f_{1 k}^{i}\left(x_{1}\right)\right] b_{k} x_{2 k}^{i} \\
& +a_{k} x_{1 k}^{r} b_{k}\left[F_{2 k}^{r}\left(x_{2}\right) P_{2}+f_{2 k}^{r}\left(x_{2}\right)\right] \\
& -a_{k} x_{1 k}^{i} b_{k}\left[F_{2 k}^{i}\left(x_{2}\right) P_{2}+f_{2 k}^{i}\left(x_{2}\right)\right]-G_{k}^{r}(y) Q \\
& -g_{k}^{r}(y)+l e_{k}^{r}
\end{aligned}
$$

$$
\begin{aligned}
& +j\left\{a_{k}\left[F_{1 k}^{r}\left(x_{1}\right) P_{1}+f_{1 k}^{r}\left(x_{1}\right)\right] b_{k} x_{2 k}^{i}\right. \\
& +a_{k}\left[F_{1 k}^{i}\left(x_{1}\right) P_{1}+f_{1 k}^{i}\left(x_{1}\right)\right] b_{k} x_{2 k}^{r} \\
& +a_{k} x_{1 k}^{r} b_{k}\left[F_{2 k}^{i}\left(x_{2}\right) P_{2}+f_{2 k}^{i}\left(x_{2}\right)\right] \\
& +a_{k} x_{1 k}^{i} b_{k}\left[F_{2 k}^{r}\left(x_{2}\right) P_{2}+f_{2 k}^{r}\left(x_{2}\right)\right]-G_{k}^{i}(y) Q \\
& \left.-g_{k}^{i}(y)+l e_{k}^{i}\right\}
\end{aligned}
$$

then the drive complex systems (10) and (11) will achieve a novel type of function projective synchronization with the response complex system (13), where $l>0$ is a constant.

Corollary 9. Assume the scaling matrices $A=0$ or $B=C=0$; in other words, the scaling matrices are the real matrices; if the control laws are chosen as

$$
\begin{aligned}
v_{k}= & -G_{k}^{r}(y) Q-g_{k}^{r}(y)+l e_{k}^{r} \\
& +j\left(-G_{k}^{i}(y) Q-g_{k}^{i}(y)+l e_{k}^{i}\right),
\end{aligned}
$$

then the equilibrium point of response system (13) is asymptotically stable.

Remark 10. In this paper, the effectiveness of the controller in terms of robustness to perturbations or discussion of stability for ranges and uncertainty in parameter values is little considered. With the development of science and technology, more uncertain factors appear to make the secure communication problem more complex and difficult to predict, which is a challenging problem in secure communication. How the method can be actually implemented in real secure communication is our future research direction.

\section{Compound Synchronization Examples}

For the convenience of our discussions, four identical chaotic complex systems are investigated to realize the compound synchronization behavior in the section. Therefore, three drive systems are given as follows:

$$
\begin{aligned}
& \dot{x}_{11}=a x_{12} x_{13}, \\
& \dot{x}_{12}=1+b x_{11}, \\
& \dot{x}_{13}=x_{11} \bar{x}_{11}+c x_{13} ; \\
& \dot{x}_{21}=a x_{22} x_{23}, \\
& \dot{x}_{22}=1+b x_{21}, \\
& \dot{x}_{23}=x_{21} \bar{x}_{21}+c x_{23} ; \\
& \dot{x}_{31}=a x_{32} x_{33}, \\
& \dot{x}_{32}=1+b x_{31}, \\
& \dot{x}_{33}=x_{31} \bar{x}_{31}+c x_{33} .
\end{aligned}
$$


The corresponding response system is as follows:

$$
\begin{aligned}
& \dot{y}_{1}=a y_{2} y_{3}+v_{1}+j v_{2}, \\
& \dot{y}_{2}=1+b y_{1}+v_{3}+j v_{4}, \\
& \dot{y}_{3}=y_{1} \bar{y}_{1}+c y_{3}+v_{5} .
\end{aligned}
$$

The feedback control law can be designed as follows:

$$
\begin{aligned}
v_{1}+ & j v_{2} \\
= & a a_{1} x_{12} x_{13}\left(b_{1} x_{21}+c_{1} x_{31}\right) \\
& +a_{1} x_{11}\left(b_{1} a x_{22} x_{23}+c_{1} a x_{32} x_{33}\right)-a y_{2} y_{3}+l e_{1}^{r} \\
& +j l e_{1}^{i}, \\
v_{3}+ & j v_{4} \\
= & a_{2}\left(1+b x_{11}\right)\left(b_{2} x_{22}+c_{2} x_{32}\right) \\
& +a_{2} x_{12}\left[b_{2}\left(1+b x_{21}\right)+c_{2}\left(1+b x_{31}\right)\right]-1-b y_{1} \\
& +l e_{2}^{r}+j l e_{2}^{i}, \\
v_{5} & \\
= & a_{3}\left(x_{11} \bar{x}_{11}+c x_{13}\right)\left(b_{3} x_{23}+c_{2} x_{33}\right) \\
& +a_{3} x_{13}\left[b_{3}\left(x_{21} \bar{x}_{21}+c x_{23}\right)+c_{3}\left(x_{31} \bar{x}_{31}+c x_{33}\right)\right] \\
& -y_{1} \bar{y}_{1}-c y_{3}+l e_{3}^{r} .
\end{aligned}
$$

To verify the feasibility of compound synchronization scheme, the simulation results of compound synchronization are investigated for four identical chaotic complex systems (26)-(29). In the numerical simulations, the parameters are chosen as $a=1, b=-4, c=-1$, and the initial values of the drive systems and the response systems are randomly chosen as $\left(x_{11}(0), x_{12}(0), x_{13}(0)\right)^{T}=$ $(1+j, 1+j, 1)^{T},\left(x_{21}(0), x_{22}(0), x_{23}(0)\right)^{T}=(2+2 j, 2+$ $2 j, 2)^{T},\left(x_{31}(0), x_{32}(0), x_{33}(0)\right)^{T}=(3+3 j, 3+3 j, 3)^{T}$, $\left(y_{1}(0), y_{2}(0), y_{3}(0)\right)^{T}=(1+j, 1+j, 1)^{T}$, respectively. The scaling complex matrices and the constant $l$ are taken as $A=B=C=\operatorname{diag}(1,1,1)$ and $l=0.6$, respectively. The corresponding numerical results are shown in Figures 1014. Figures 10-14 show the mixed system of drive systems (26), (27), and (28) can synchronize the response system (29), respectively. As one can see, synchronization errors $e_{i}(1,2,3,4,5)$ of time response have converged to zero, as $t \rightarrow 0$, which implies that systems (26)-(29) have achieved the compound synchronization, where $\operatorname{Re}(\cdot)$ and $\operatorname{Im}(\cdot)$ stand for real and imaginary parts of the complex variables.

Remark 11. In the simulation, since the third variables are the real variables for chaotic complex systems (26)-(29), we do not consider the imaginary part of the third variable. The design of controllers $v_{16}$ and $v_{26}$ is not considered to eliminate the influence of imaginary part. Hence, the compound synchronization of the third variable is limited to the real part of the third variable for chaotic complex systems

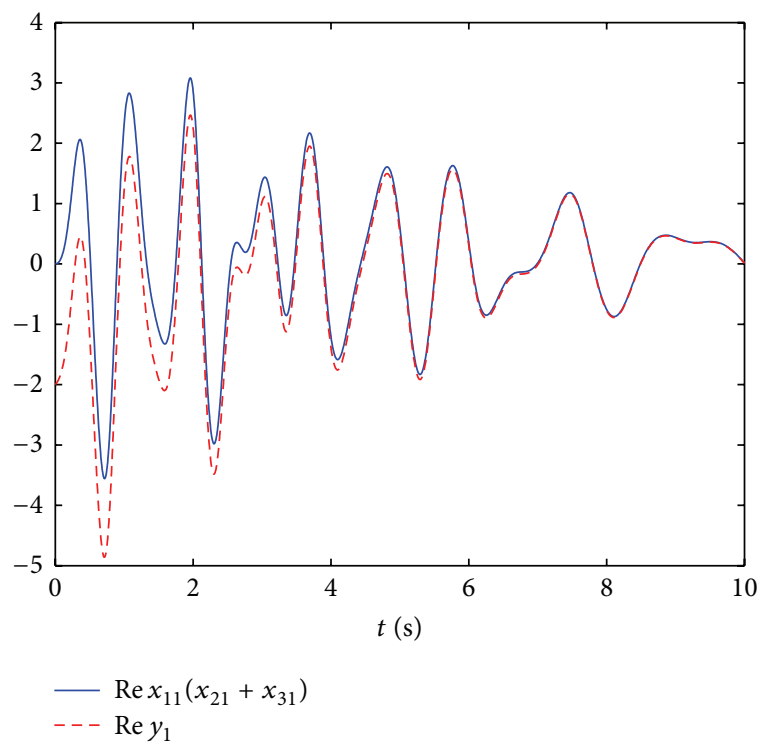

Figure 10: Compound synchronization of real part of the first variable.

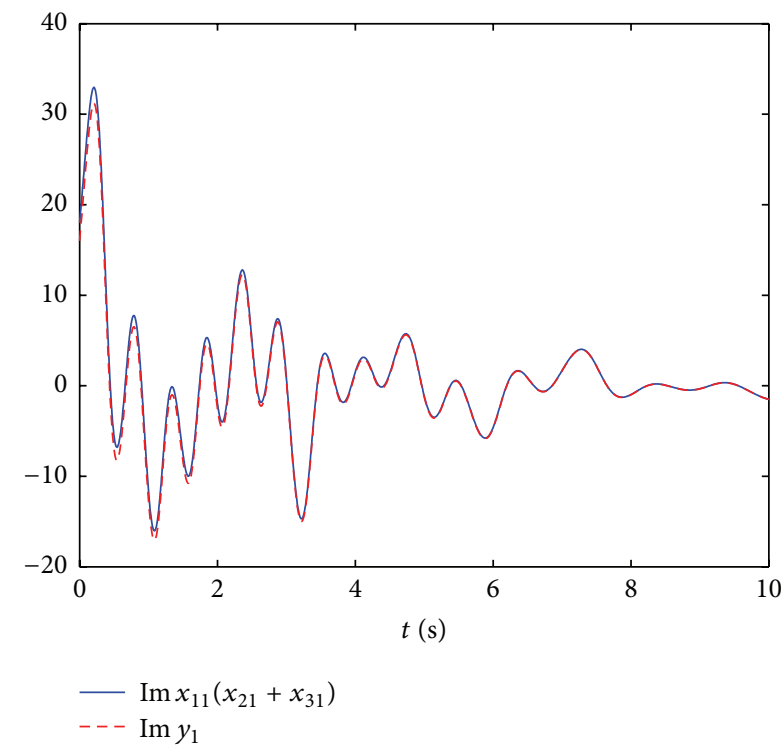

FIGURE 11: Compound synchronization of imaginary part of the first variable.

(26)-(29) as Figure 14, which can save a lot of time and energy for our future applications.

\section{Conclusion}

A novel chaotic complex system has been designed in this paper, whose basic dynamical properties of chaotic complex system including invariance, dissipativity, equilibria, Lyapunov exponents, bifurcation diagrams, chaotic behavior, and chaotic attractors have been discussed. Based on compound synchronization of four chaotic real systems, a general scheme of compound synchronization among four chaotic complex systems has been investigated with regard to the 


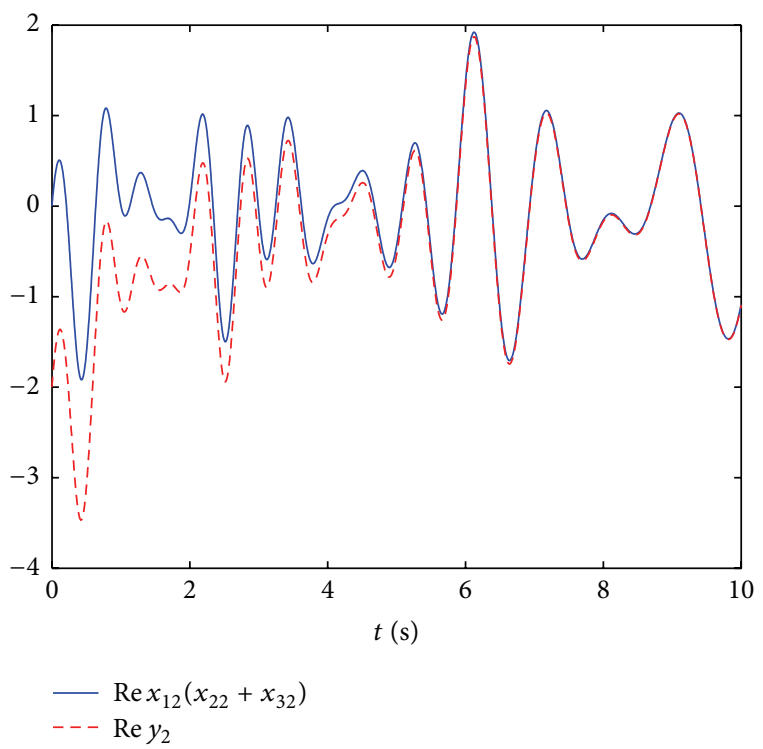

FIGURE 12: Compound synchronization of real part of the second variable.

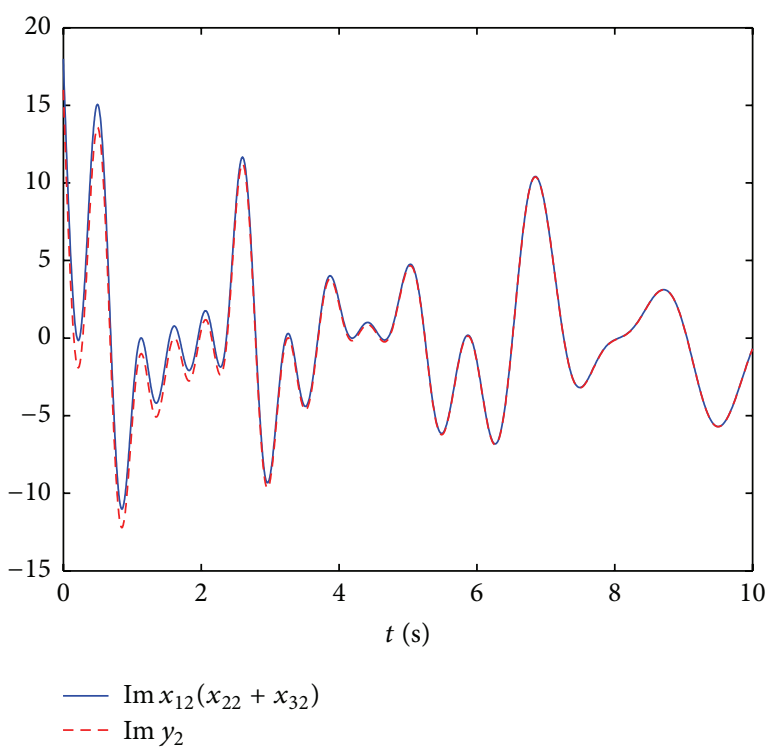

FIGURE 13: Compound synchronization of imaginary part of the second variable.

real scaling matrices. According to Lyapunov stability theory, some sufficient conditions for compound synchronization of four chaotic complex systems have been gained via the adaptive control technique. Four novel chaotic complex systems are considered and the corresponding controllers have been given as an example to illustrate the effectiveness of the proposed scheme.

\section{Conflict of Interests}

The authors declare that there is no conflict of interests regarding the publication of this paper.

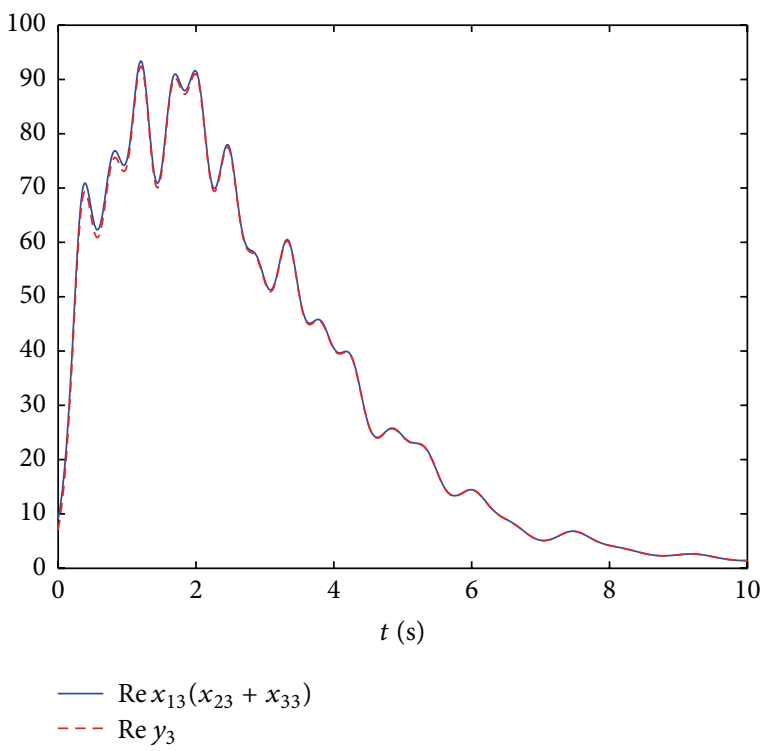

FIGURE 14: Compound synchronization of real part of the third variable.

\section{Acknowledgments}

The authors thank the editor and the anonymous reviewers for their resourceful and valuable comments and constructive suggestions. The work is supported by the State Key Program of the National Natural Science Foundation of China (Grant no. 61134012), the National Natural Science Foundation of China (Grant nos. 61472371, 61472372, and 61572446), China Postdoctoral Science Foundation funded project (Grant no. 2015M570641), and the Science Foundation of Doctorate Research of Zhengzhou University of Light Industry (Grant no. 2014BSJJ044).

\section{References}

[1] L. M. Pecora and T. L. Carroll, "Synchronization in chaotic systems," Physical Review Letters, vol. 64, no. 8, pp. 821-824, 1990.

[2] A. Wu, S. Wen, and Z. Zeng, "Synchronization control of a class of memristor-based recurrent neural networks," Information Sciences, vol. 183, no. 1, pp. 106-116, 2012.

[3] Y. Gao, X. Zhang, and G. Lu, "Dissipative synchronization of nonlinear chaotic systems under information constraints," Information Sciences, vol. 225, pp. 81-97, 2013.

[4] S.-Y. Li, H.-K. Chen, L.-M. Tam, S.-C. Huang, and Z.-M. Ge, "Pragmatical adaptive synchronization-new fuzzy model of two different and complex chaotic systems by new adaptive control," Information Sciences, vol. 277, pp. 458-480, 2014.

[5] P. P. Singh, J. P. Singh, and B. K. Roy, "Synchronization and antisynchronization of Lu and Bhalekar-Gejji chaotic systems using nonlinear active control," Chaos, Solitons \& Fractals, vol. 69, pp. 31-39, 2014.

[6] T. Song, L. Pan, and G. Păun, "Asynchronous spiking neural P systems with local synchronization," Information Sciences, vol. 219, pp. 197-207, 2013. 
[7] W. He and J. Cao, "Generalized synchronization of chaotic systems: an auxiliary system approach via matrix measure," Chaos, vol. 19, no. 1, Article ID 013118, 2009.

[8] L. Kocarev and U. Parlitz, "Generalized synchronization, predictability, and equivalence of unidirectionally coupled dynamical systems," Physical Review Letters, vol. 76, no. 11, pp. 18161819, 1996.

[9] E. M. Shahverdiev and K. A. Shore, "Generalized synchronization in time-delayed systems," Physical Review E, vol. 71, no. 1, Article ID 016201, 2005.

[10] P. K. Roy, C. Hens, I. Grosu, and S. K. Dana, "Engineering generalized synchronization in chaotic oscillators," Chaos, vol. 21, no. 1, Article ID 013106, 2011.

[11] X. Wu, J. Cai, and M. Wang, "Robust synchronization of chaotic horizontal platform systems with phase difference," Journal of Sound and Vibration, vol. 305, no. 3, pp. 481-491, 2007.

[12] M.-C. Ho, Y.-C. Hung, and C.-H. Chou, "Phase and anti-phase synchronization of two chaotic systems by using active control," Physics Letters A, vol. 296, no. 1, pp. 43-48, 2002.

[13] G.-P. Jiang, W. X. Zheng, and G. Chen, "Global chaos synchronization with channel time-delay," Chaos, Solitons and Fractals, vol. 20, no. 2, pp. 267-275, 2004.

[14] C. Li, X. Liao, and K.-W. Wong, "Lag synchronization of hyperchaos with application to secure communications," Chaos, Solitons and Fractals, vol. 23, no. 1, pp. 183-193, 2005.

[15] Z.-M. Ge and Y.-S. Chen, "Synchronization of unidirectional coupled chaotic systems via partial stability," Chaos, Solitons and Fractals, vol. 21, no. 1, pp. 101-111, 2004.

[16] H. Du, Q. Zeng, and C. Wang, "Modified function projective synchronization of chaotic system," Chaos, Solitons and Fractals, vol. 42, no. 4, pp. 2399-2404, 2009.

[17] J. Sun, Y. Shen, and G. Zhang, "Transmission projective synchronization of multi-systems with non-delayed and delayed coupling via impulsive control," Chaos, vol. 22, no. 4, Article ID 043107, 2012.

[18] A. E. Hramov and A. A. Koronovskii, "Time scale synchronization of chaotic oscillators," Physica D: Nonlinear Phenomena, vol. 206, no. 3-4, pp. 252-264, 2005.

[19] J. Sun, Y. Shen, X. Wang, and J. Chen, "Finite-time combinationcombination synchronization of four different chaotic systems with unknown parameters via sliding mode control," Nonlinear Dynamics, vol. 76, no. 1, pp. 383-397, 2014.

[20] L. Runzi and W. Yinglan, "Active backstepping-based combination synchronization of three different chaotic systems," Advanced Science, Engineering and Medicine, vol. 4, no. 2, pp. 142-147, 2012.

[21] J. Sun, Y. Shen, G. Zhang, C. Xu, and G. Cui, "Combinationcombination synchronization among four identical or different chaotic systems," Nonlinear Dynamics, vol. 73, no. 3, pp. 12111222, 2013.

[22] J. Sun, Y. Shen, Q. Yin, and C. Xu, "Compound synchronization of four memristor chaotic oscillator systems and secure communication," Chaos, vol. 23, no. 1, Article ID 013140, 2013.

[23] J. Sun, Q. Yin, and Y. Shen, "Compound synchronization for four chaotic systems of integer order and fractional order," $E P L$, vol. 106, no. 4, Article ID 40005, 2014.

[24] A. C. Fowler, J. D. Gibbon, and M. J. McGuinness, "The complex Lorenz equations," Physica D: Nonlinear Phenomena, vol. 4, no. 2, pp. 139-163, 1982.

[25] J. Sun and Y. Shen, "Quasi-ideal memory system," IEEE Transactions on Cybernetics, vol. 45, pp. 1353-1362, 2015.
[26] G. M. Mahmoud, T. Bountis, and E. E. Mahmoud, "Active control and global synchronization of the complex chen and Lü systems," International Journal of Bifurcation and Chaos, vol. 17, no. 12, pp. 4295-4308, 2007.

[27] G. M. Mahmoud, E. E. Mahmoud, and M. E. Ahmed, "On the hyperchaotic complex Lü system," Nonlinear Dynamics, vol. 58, no. 4, pp. 725-738, 2009.

[28] G. M. Mahmoud, S. A. Aly, and M. A. Al-Kashif, "Dynamical properties and chaos synchronization of a new chaotic complex nonlinear system," Nonlinear Dynamics, vol. 51, no. 1-2, pp. 171$181,2008$.

[29] G. M. Mahmoud and E. E. Mahmoud, "Phase and antiphase synchronization of two identical hyperchaotic complex nonlinear systems," Nonlinear Dynamics, vol. 61, no. 1-2, pp. 141-152, 2010.

[30] G. M. Mahmoud and E. E. Mahmoud, "Complete synchronization of chaotic complex nonlinear systems with uncertain parameters," Nonlinear Dynamics, vol. 62, no. 4, pp. 875-882, 2010.

[31] J. C. Sprott, "Some simple chaotic flows," Physical Review E, vol. 50, no. 2, pp. R647-R650, 1994. 


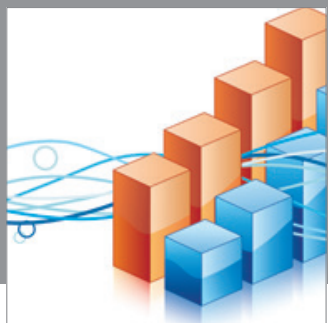

Advances in

Operations Research

mansans

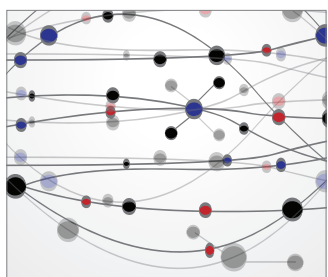

The Scientific World Journal
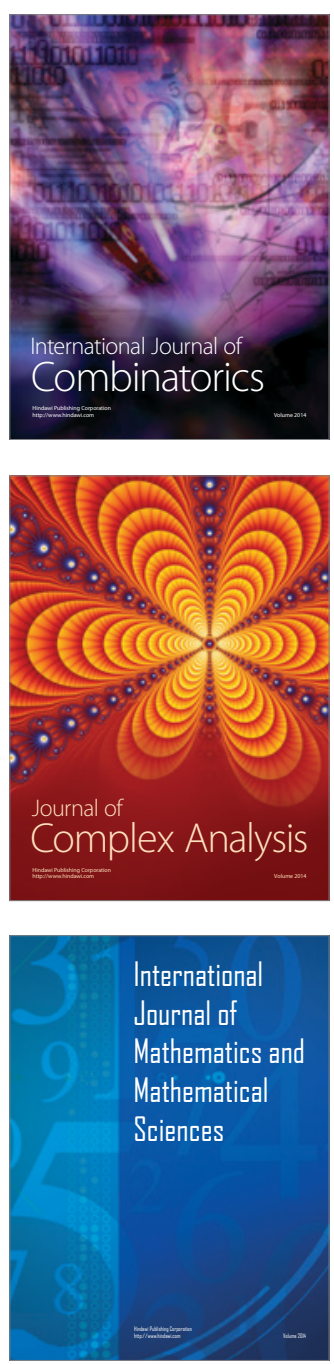
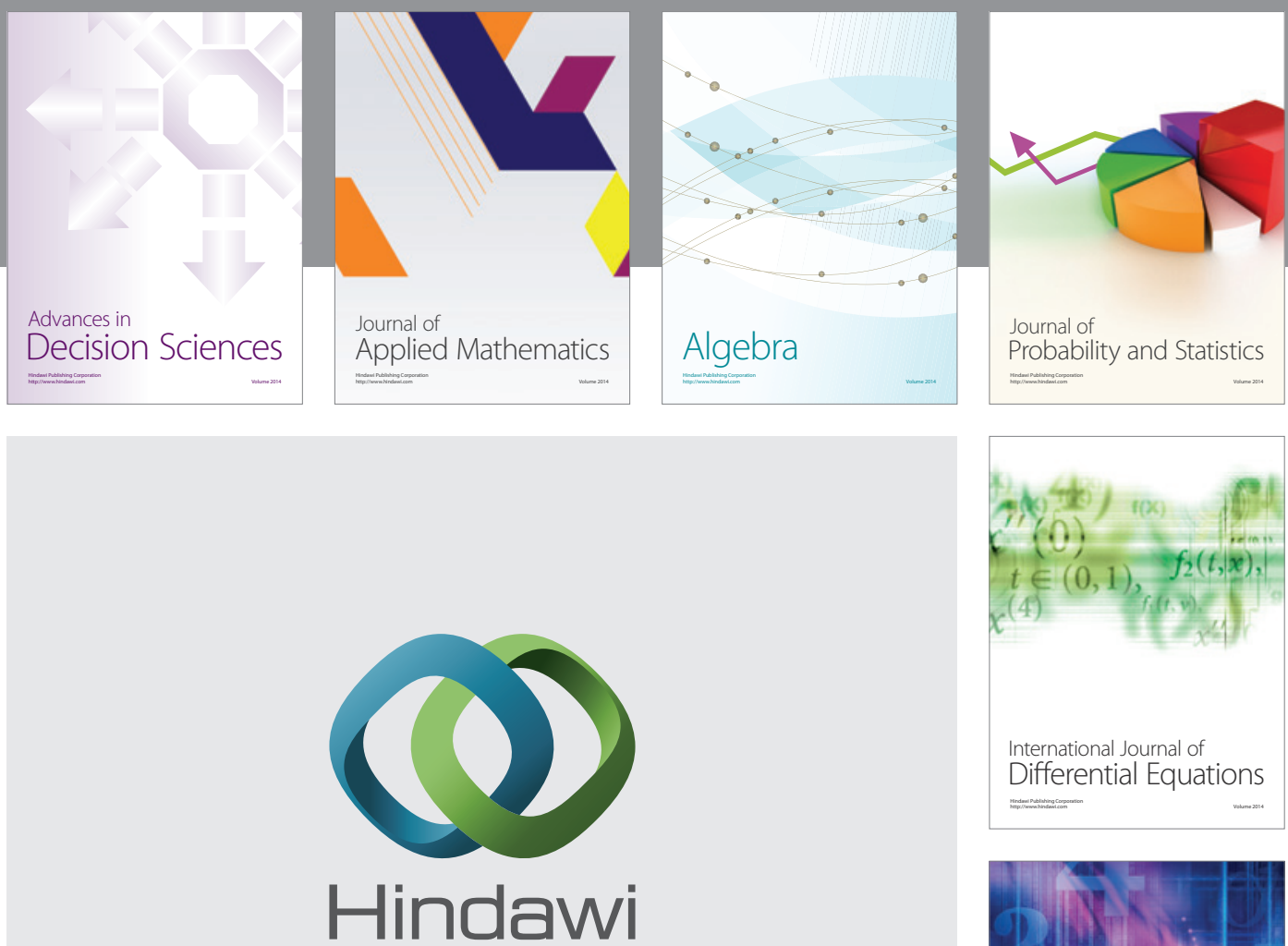

Submit your manuscripts at http://www.hindawi.com
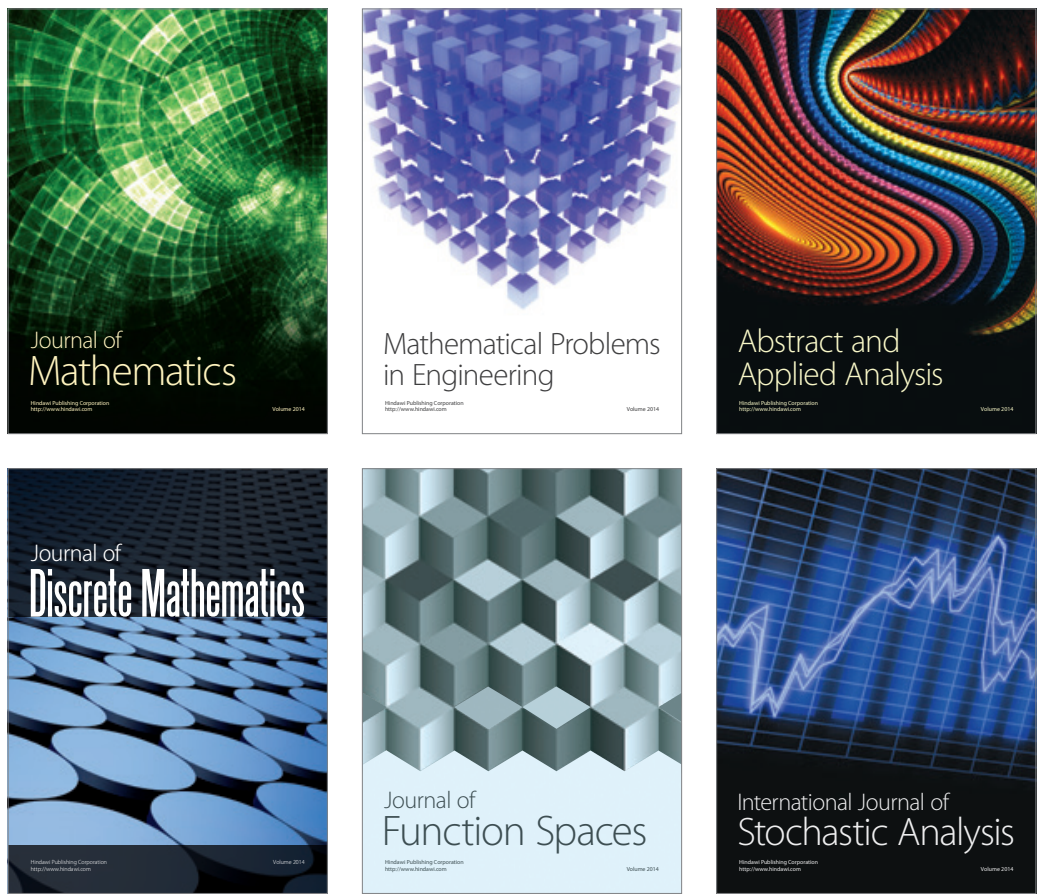

Journal of

Function Spaces

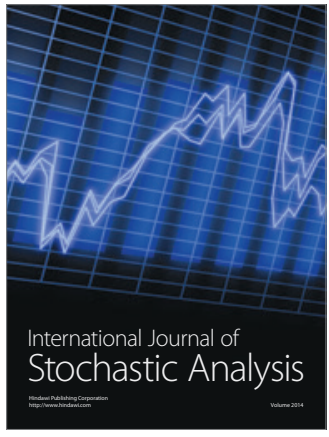

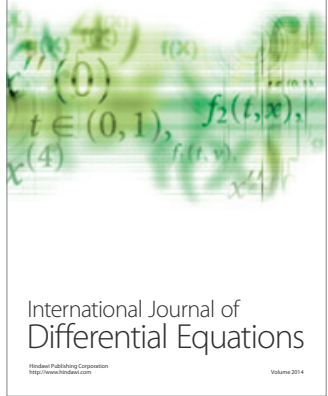
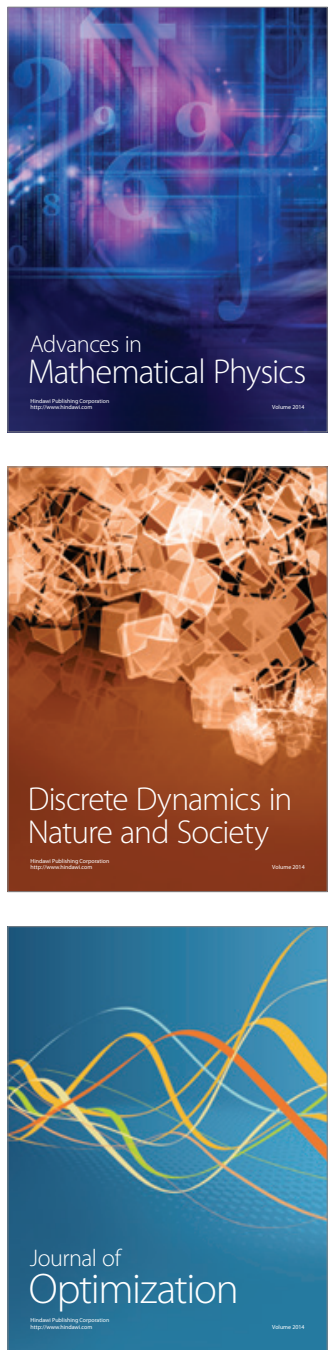